\title{
Self-consistent one-dimensional electron system on liquid helium suspended over a nanoscale dielectric substrate
}

\author{
Oleg G. Balev ${ }^{\mathrm{a}, *}$, Antonio C.A. Ramos ${ }^{\mathrm{b}}$ \\ ${ }^{a}$ Departamento de Física, Universidade Federal do Amazonas, 69077-000, Manaus, Amazonas, Brazil \\ ${ }^{b}$ Grupo de Fúsica Teórica e Computacional, Universidade Federal do Cariri, 63048-080, Juazeiro do Norte, Ceará, Brazil
}

\section{Abstract}

For electrons above a superfluid helium film suspended on a specially designed dielectric substrate, $z=h(y)$, we obtain that both the transverse, along $z$, and the lateral, along $y$, quantizations are strongly enhanced due to a strong mutual 'coupling. The self-consistent quantum wires (QWs) with non-degenerated one-dimensional electron systems (1DESs) are obtained over a superfluid liquid helium (LH) suspended self-consistently on different dielectric substrates with a nanoscale modulation. A gap $\gtrsim 10 \mathrm{meV}(\gtrsim 1 \mathrm{meV})$ is obtained between the lowest two electron levels due to mainly the transverse (lateral) quantization. Our analytical model takes into account a strong interplay between the transverse and the lateral quantizations of an electron. It uses that the characteristic length (energy) along the former direction is essentially smaller (larger) than the one along the latter, in a close analogy with the adiabatic approximation.

Keywords: self-consistent, quantum wire, electronic structure, nanoscale system, quantum computing

PACS: 68.15.+e, 67.25.bh, 67.25.D, 73.22.-f, 03.67.Lx

\section{Introduction}

Since pioneering works [1 $[\underline{3}]$ quantized states of electrons above LH suspended on different substrates are the subject of a strong ongoing interest [4 23$]$. Electrons floating on LH have been proposed for quantum computing in a seminal work Ref. [10]. For a plane substrate and a large thickness of LH film, $d \gtrsim 0.5 \mu \mathrm{m}$, any effect of the substrate is negligible [4, 6]. This allows a two-dimensional electron system (2DES) on a bulk LH and a single electron on a bulk film [10] with a $1 \mathrm{D}$ hydrogenic spectrum [4 [ 6, 10] $E_{m}^{1 H}=-R / m^{2}$. Here $R \approx 8 \mathrm{~K}$ is an effective Rydberg energy. For quantum computing in Ref. [10] it is suggested to patten the bottom electrode with features spaced close to $d(\approx 0.5 \mu \mathrm{m})$. So that each feature traps one electron. Metallic posts submerged by the depth $\sim 0.5 \mu \mathrm{m}$, beneath practically plane helium surface, are suggested [12]. They form quantum dots for electrons on LH which may serve as the qubits of a quantum computer [12], in particular, at temperature $T \approx 10 \mathrm{mK}[10,12]$. Surface electrons with band-type spectrum on LH over metallic periodic substrate of the diffraction grating type are proposed by Ginzburg and Monarkha 7]. Where an amplitude of modulation is much smaller than $d$ and a free surface of LH is assumed as flat.

Electrons in a micron-scale and a nanoscale channels filled by capillary action with LH $[\underline{5}, \underline{8}-10,13,23]$ attract

\footnotetext{
* Corresponding author

Email addresses: ogbalev@ufam.edu.br (Oleg G. Balev), antonio.ramos@ufca.edu.br (Antonio C.A. Ramos)
}

recently much attention, in particular, due to their high potential in creating qubits with the needed properties of performance. The systems of such channels are promising for construction of the equivalent of a charge-coupled device (CCD) 24] that, in addition, will allow the large scale transport of qubits [15, 16, 19]. In interesting experiments of Refs. [16, 19] electrons are studied in the channels of a width $\gtrsim 3 \mu \mathrm{m}$ at $T \approx 1.5 \mathrm{~K}$. Theoretical framework of Refs. [16, 19] treats electrons mainly as ones above a bulk LH.

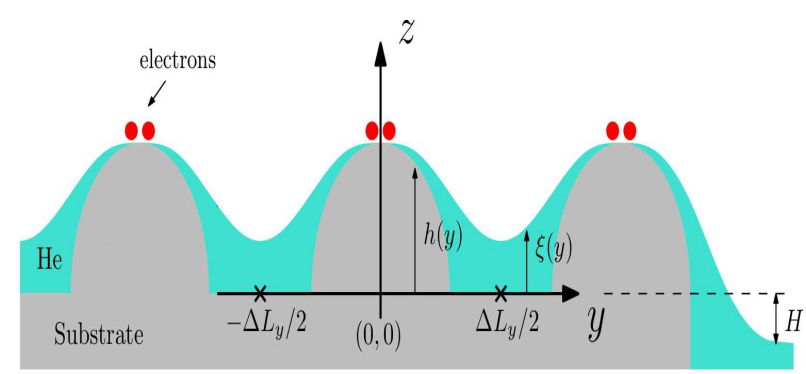

Figure 1: (Color online) A sketch, not to scale, of a model geometry. Substrate surface $z=h(y)$, LH surface $z=\xi(y)$, and LH thickness $d(y)=\xi(y)-h(y)$

Indeed, usually some important characteristics of electrons in these channels such as a gap between the lowest electron levels of the transverse (lateral) quantization, a form of the lateral potential, a form of the LH surface, a lateral density profile, etc. are not well known [5, 8, 9, 1417, 19 23. In particular, due to absence of interplay and self-consistency between transverse and lateral quantiza- 
tions within used theoretical frameworks.

In present study self-consistent 1DESs in QWs over LH suspended on different nanoscale dielectric substrates are obtained, for $T=0.6 \mathrm{~K}$. A strong interplay between the quantizations of an electron along $z$ and $y$ directions is treated within present approach. It uses, in particular, some analogies with well known adiabatic approximation [25]. In Fig. 1 the sketch of a geometry of studied model is shown. We consider that a dielectric substrate is periodic along the $y$-direction with a finite period $\Delta L_{y}$ (unless otherwise stated) and $L_{x} \rightarrow \infty$. Within the main super cell $\left(L_{x} \times \Delta L_{y} ;|y| \leq \Delta L_{y} / 2\right)$ the substrate profile $z=h(y)$ is assumed as

$$
\begin{aligned}
& h(y)=0, \quad a / 4 \leq|y| \leq \Delta L_{y} / 2, \\
& h(y)=h_{1} \cos (2 \pi y / a), \quad|y| \leq a / 4,
\end{aligned}
$$

where $h_{1}, a, \Delta L_{y}$ are the characteristic scales of its modulation, cf. Fig. 11 We assume that $2 h_{1} / a \ll 1$. I.e., a substrate profile is smooth.

For obtained systems of QWs, within the main super cell a 1DES is laterally localized at $y=0$, cf. Fig. 1. Point out that effect of tunnel coupling between 1DESs of neighboring super cells is negligible for present systems of QWs. We have obtained a strong "long-range" effect of $\Delta L_{y}$ on the properties of a self-consistent 1DES at the region $10 \mu \mathrm{m} \geq \Delta L_{y} \geq 1 \mu \mathrm{m}$. For a given linear density within a super cell $n_{L}=N_{t o t} / L_{x} ; N_{t o t}$ is the total number of electrons within a super cell. It is related with an essential dependence of LH profile within this region. That induces a strong modification of the transverse and the lateral quantizations for an electron. In particular, an essential modification of the effective electron potential is obtained due to a strong change for the image potential of substrate.

Notice, for $\Delta L_{y} \gtrsim 50 \mu \mathrm{m}$ properties of a self-consistent 1DES become practically independent of $\Delta L_{y}$. In present figures we assume that $\Delta L_{y}=1 \mu \mathrm{m}$ or $10 \mu \mathrm{m}$. Then obtained results can be applied to the properties of QWs within a finite region $|y| \leq L_{y} / 2$, with $L_{y} \geq \Delta L_{y}$, if the substrate have a finite region of periodic modulation $|y| \geq L_{y} / 2+25 \mu \mathrm{m}$.

In Subsection 2.1 we present a self-consistent Hamiltonian of an electron on a self-consistent LH film, suspended over a dielectric substrate with a nanoscale lateral modulation. In Subsection 2.2 we give the rest of a self-consistent framework for our model. It defines a self-consistent profile of LH suspended on the dielectric substrate, for a given linear density within a super cell. In Section 3 we present results and discussions on the self-consistent profiles of $\mathrm{LH}$ films suspended on the special dielectric substrates, the lowest levels of the transverse and of the lateral quantizations, a self-consistent electron density $n(y)$ profiles of 1DESs in obtained self-consistent electron nano-channels. Conclusions follow in Section 4.

\section{Self-consistent model of electrons over liquid he- lium on a substrate with nanoscale modulation}

\subsection{One-electron Hamiltonian}

We consider that between a surface of LH, $z=\xi(y)$, and the surface of substrate, $z=h(y)$, Eq. (11) a LH film is formed of the thickness $d(y)=\xi(y)-h(y)>0$. First we assume that $\Delta L_{y} \rightarrow \infty$, later on we will show how our study can be extended to a finite $\Delta L_{y}$. Then the wave functions and the eigenvalues of an electron over LH are defined by the Schrodinger equation [5, 6, 14]

$$
\begin{aligned}
& {\left[-\frac{\hbar^{2}}{2 m_{0}}\left(\frac{\partial^{2}}{\partial z^{2}}+\frac{\partial^{2}}{\partial y^{2}}+\frac{\partial^{2}}{\partial x^{2}}\right)+V(z, y)\right]} \\
& \times \Psi_{\beta}(z, y, x)=W_{\beta} \Psi_{\beta}(z, y, x),
\end{aligned}
$$

where three quantum numbers $\beta=\left\{k_{x \beta}, n_{y \beta}, n_{z \beta}\right\}$ are given by the wave number $k_{x \beta}=2 \pi n_{x \beta} / L_{x}$ and two integer quantum numbers $n_{y \beta}=1,2,3, \ldots, n_{z \beta}=1,2,3, \ldots$. As we assume the Born-von Karman boundary condition along $x$, we have $n_{x \beta}=0, \pm 1, \pm 2, \ldots$ In Eq. (2), e.g., following Refs. [5, 6, 14], we have that

$$
V(z, y)=-\frac{\Lambda}{z-\xi(y)}-\frac{\Lambda_{1}}{z-h(y)}+|e| E_{p} z,
$$

where $\Lambda=e^{2}\left(\varepsilon_{L H}-1\right) /\left[4\left(\varepsilon_{L H}+1\right)\right]$ and $\Lambda_{1}=e^{2}\left(\varepsilon_{S}-\right.$ $1) /\left[4\left(\varepsilon_{S}+1\right)\right]$. Here $\varepsilon_{L H} \approx 1.054$ is the dielectric constant of LH, $\varepsilon_{S}$ is the dielectric constant of substrate, and $E_{p}$ is an external (also called as holding) electric field. The first two terms in the right hand side of Eq. (3) represent the main contributions to the image potential energy [6]. The former term represents the image potential energy due to a bulk LH and the latter one shows a main effect of the substrate (for an infinite thickness of a LH film it is nullified).

Point out, Eq. (3) can be considered as exact if $\xi(y)$ and $h(y)$ are the linear polynomial functions of $y$ or independent of $y$. For more complex dependences of $\xi(y)$ and $h(y)$ on $y$, Eq. (3) is valid if $h(y)$ is smooth enough within an actual region. Where an electron is present mainly. This justifies the second term in Eq. (3). Further, the first term in Eq. (3) is readily justified due to a smoother $\xi(y)$ than $h(y)$ and closer average position of an electron along $z$ to the characteristic boundary. Here it is the LH surface $\xi(y)$. I.e., in Eq.(3) an electron image potential is well approximated by the first two terms of the right hand side provided the distance between the electron and the dielectric is small relative to the curvature of the dielectric surface.

As potential Eq. (3) is independent of $x$ we look for a solution of Eq. (2) as follows

$$
\Psi_{\beta}(z, y, x)=L_{x}^{-1 / 2} e^{i k_{x \beta} x} \psi_{n_{z \beta}, n_{y \beta}}(z, y) .
$$

Then from Eq. (2) we obtain

$$
\begin{aligned}
& {\left[-\frac{\hbar^{2}}{2 m_{0}}\left(\frac{\partial^{2}}{\partial z^{2}}+\frac{\partial^{2}}{\partial y^{2}}\right)+V(z, y)\right] \psi_{n_{z \beta}, n_{y \beta}}(z, y)} \\
& =\widetilde{W}_{n_{z \beta}, n_{y \beta}} \psi_{n_{z \beta}, n_{y \beta}}(z, y),
\end{aligned}
$$


where $\widetilde{W}_{n_{z \beta}, n_{y \beta}}=W_{\beta}-\frac{\hbar^{2} k_{x \beta}^{2}}{2 m_{0}}$.

To solve Eq. (5) we develop an approach similar with the well known adiabatic method [25], that separates a fast movement of electrons from a slow movement of nuclei, to separate a fast movement along z-axis, on a short space scale $\Delta z$, from a slow movement along y-axis, on the scale $\Delta y \gg \Delta z$. We assume that

$$
\psi_{n_{z \beta}, n_{y \beta}}(z, y)=\Phi_{n_{y \beta}}(y) \varphi_{n_{z \beta}}(z, y),
$$

where $\varphi_{n_{z \beta}}(z, y)$ is a real function (this condition always can be satisfied as it is a discrete spectrum state; $n_{z \beta}=$ $1,2, \ldots)$ that satisfies

$$
\left[-\frac{\hbar^{2}}{2 m_{0}} \frac{\partial^{2}}{\partial z^{2}}+V(z, y)\right] \varphi_{n_{z \beta}}(z, y)=\mathcal{E}_{n_{z \beta}}(y) \varphi_{n_{z \beta}}(z, y),
$$

where $y$ has the role of a parameter. Then, substituting Eq. (6) in Eq. (5) and using Eq. (77), we obtain

$$
\begin{aligned}
& -\frac{\hbar^{2}}{2 m_{0}}\left[\varphi_{n_{z \beta}}(z, y) \frac{\partial^{2}}{\partial y^{2}} \Phi_{n_{y \beta}}(y)+2 \frac{\partial \Phi_{n_{y \beta}}(y)}{\partial y}\right. \\
& \left.\times \frac{\partial \varphi_{n_{z \beta}}(z, y)}{\partial y}+\Phi_{n_{y \beta}}(y) \frac{\partial^{2}}{\partial y^{2}} \varphi_{n_{z \beta}}(z, y)\right] \\
& =\left(\widetilde{W}_{n_{z \beta}, n_{y \beta}}-\mathcal{E}_{n_{z \beta}}(y)\right) \Phi_{n_{y \beta}}(y) \varphi_{n_{z \beta}}(z, y) .
\end{aligned}
$$

As a wave function of discrete spectrum $\varphi_{n_{z \beta}}(z, y)=0$, for $z \leq \xi(y)$, and it is localised at $z \approx \xi(y)$ (e.g., within a few nanometers from the LH surface for typical conditions of below Figs. 2-11), we obtain from its normalization

$$
\int_{-\infty}^{\infty} d z \varphi_{n_{z \beta}}^{2}(z, y)=1
$$

after applying $\partial / \partial y$, that

$$
\int_{-\infty}^{\infty} d z \varphi_{n_{z \beta}}(z, y) \partial \varphi_{n_{z \beta}}(z, y) / \partial y=0 .
$$

Then multiplying Eq. (8) by $\varphi_{n_{z \beta}}(z, y)$ and integrating over $\mathrm{z}, \int_{-\infty}^{\infty} d z$, and using Eqs. (9)-(10), we obtain

$$
\begin{aligned}
& -\frac{\hbar^{2}}{2 m_{0}} \frac{d^{2}}{d y^{2}} \Phi_{n_{y \beta}}(y)+\left[\mathcal{E}_{n_{z \beta}}(y)-\frac{\hbar^{2}}{2 m_{0}}\right. \\
& \left.\times \int_{-\infty}^{\infty} d z \varphi_{n_{z \beta}}(z, y) \frac{\partial^{2}}{\partial y^{2}} \varphi_{n_{z \beta}}(z, y)\right] \Phi_{n_{y \beta}}(y) \\
& =\widetilde{W}_{n_{z \beta}, n_{y \beta}} \Phi_{n_{y \beta}}(y) .
\end{aligned}
$$

Using Eq. (77) and $(\Delta z / \Delta y)^{2} \ll 1$, we assume that the second term in the square brackets of Eq. (11) gives only a small correction to the first term, $\mathcal{E}_{n_{z \beta}}(y)$, and can be neglected. More accurate condition of a smallness of the non-adiabatic term in Eq. (11) is given below by Eq. (22). In particular, for studied in Figs 2-11]self-consistent quantum wires over LH non-adiabatic contributions in Eq. (11) are very small. Then, similar with the adiabatic approximation, we have

$$
\begin{aligned}
& -\frac{\hbar^{2}}{2 m_{0}} \frac{d^{2}}{d y^{2}} \Phi_{n_{y \beta}}\left(y ; n_{z \beta}\right)+\mathcal{E}_{n_{z \beta}}(y) \Phi_{n_{y \beta}}\left(y ; n_{z \beta}\right) \\
& =\widetilde{W}_{n_{z \beta}, n_{y \beta}} \Phi_{n_{y \beta}}\left(y ; n_{z \beta}\right)
\end{aligned}
$$

where $n_{z \beta}$ is a discrete parameter; usually we will be interested in the lowest level $n_{z \beta}=1$ and the first exited level $n_{z \beta}=2$ of Eq. (77). Here we show explicitly a dependence of the wave function $\Phi_{n_{y \beta}}(y)$ on $n_{z \beta}$ (cf. with Eqs. (6), (8), (11) ) as $\Phi_{n_{y \beta}}\left(y ; n_{z \beta}\right)$. Notice, it is assumed that wave functions $\Phi_{n_{y \beta}}\left(y ; n_{z \beta}\right)$ are normalized within the main super cell, $\int_{-\Delta L_{y} / 2}^{\Delta L_{y} / 2} d y\left|\Phi_{n_{y \beta}}\left(y ; n_{z \beta}\right)\right|^{2}=1$, for $\Delta L_{y} \rightarrow \infty$. However, present treatment also holds with a finite $\Delta L_{y}$ for the lowest levels $\widetilde{W}_{n_{z \beta}, n_{y \beta}}$ occupied by electrons if the tunnel coupling between such states in neighboring super cells is negligible for any realistic properties of experimental setup. In particular, as well for a very high quality setups with a very small effect of disorder on these energy levels.

Now we rewrite Eq. (77) using, instead of $z$, a new variable $\tilde{z}=z-\xi(y)$ as

$$
\begin{aligned}
& {\left[-\frac{\hbar^{2}}{2 m_{0}} \frac{\partial^{2}}{\partial \tilde{z}^{2}}+\left(-\frac{\Lambda}{\tilde{z}}-\frac{\Lambda_{1}}{\tilde{z}+d(y)}\right.\right.} \\
& \left.\left.+|e| E_{p}(\tilde{z}+\xi(y))\right)\right] \varphi_{n_{z \beta}}(\tilde{z}+\xi(y), y) \\
& =\mathcal{E}_{n_{z \beta}}(y) \varphi_{n_{z \beta}}(\tilde{z}+\xi(y), y) .
\end{aligned}
$$

Let us introduce the characteristic scales of the length $a_{0}=\hbar^{2} / \Lambda m_{0} \approx 76 \AA$, of the time $t_{0}=\hbar^{3} / m_{0} \Lambda^{2}$, of the energy $E_{0}=m_{0} \Lambda^{2} / \hbar^{2} \approx 16 \mathrm{~K}$, and a dimensionless variable $x=\tilde{z} / a_{0}$. Then Eq. (13) we rewrite, with $\varphi_{n_{z \beta}}^{y}(x)=\varphi_{n_{z \beta}}\left(a_{0} x+\xi(y), y\right)$, as

$$
\begin{aligned}
& {\left[\frac{\partial^{2}}{\partial x^{2}}+2\left(\frac{\mathcal{E}_{n_{z \beta}}(y)}{E_{0}}+\frac{1}{x}+\frac{\Lambda_{1} / \Lambda}{x+d(y) / a_{0}}\right.\right.} \\
& \left.\left.-\frac{|e| E_{p} a_{0}}{E_{0}}\left(x+\xi(y) / a_{0}\right)\right)\right] \varphi_{n_{z \beta}}^{y}(x)=0,
\end{aligned}
$$

where $\infty>x \geq 0$ and $\varphi_{n_{z \beta}}^{y}(0)=0$, as it is assumed that the wave function do not penetrate into LH. We will look for a solution of Eq. (14) using an expansion over the complete set of functions $\chi_{n}(x)$, within the interval $\infty>x \geq 0$, as

$$
\varphi_{n_{z \beta}}^{y}(x)=\sum_{n=1}^{K} C_{n_{z \beta}}^{(n)}(y) \chi_{n}(x),
$$

where a positive integer $K \rightarrow \infty$, and $\chi_{n}(x)$ satisfies the equation for radial wave functions of the hydrogen atom with zero orbital quantum number [26]

$$
\frac{d^{2}}{d x^{2}} \chi_{n}(x)=\left[\frac{1}{n^{2}}-\frac{2}{x}\right] \chi_{n}(x) .
$$

Here

$$
\int_{0}^{\infty} d x \chi_{m}(x) \chi_{n}(x)=\delta_{m, n},
$$

where $\delta_{m, n}$ is the Kronecker delta symbol, and [26]

$$
\chi_{n}(x)=\frac{2}{n^{5 / 2}} x e^{-x / n} L_{n-1}^{1}\left(\frac{2 x}{n}\right),
$$

where $L_{n-1}^{1}(2 x / n)$ is the generalized polynomial of Laguerre [27]. In present study the generalized polynomial 
of Laguerre is defined as in Ref. [27]; in Ref. [26] this polynomial has somewhat different definition.

Point out that according to Eqs. (9), (15), (17)

$$
a_{0} \int_{0}^{\infty} d x\left[\varphi_{n_{z \beta}}^{y}(x)\right]^{2}=a_{0} \sum_{n=1}^{K}\left[C_{n_{z \beta}}^{(n)}(y)\right]^{2}=1
$$

in addition, we also will use dimensionless $\widetilde{\varphi}_{n_{z \beta}}^{y}(x)=a_{0}^{1 / 2} \varphi_{n_{z \beta}}^{y}(x)$ and $\widetilde{C}_{n_{z \beta}}^{(n)}(y)=a_{0}^{1 / 2} C_{n_{z \beta}}^{(n)}(y)$ that give

$$
\int_{0}^{\infty} d x\left[\widetilde{\varphi}_{n_{z \beta}}^{y}(x)\right]^{2}=\sum_{n=1}^{K}\left[\widetilde{C}_{n_{z \beta}}^{(n)}(y)\right]^{2}=1 .
$$

Assuming that $\mathcal{E}_{n_{z \beta}=1}(y)$ has a minimum value at $y=0$ and introducing a shorter notation $W_{n_{y \beta}}=\widetilde{W}_{n_{z \beta=1}, n_{y \beta}}$ from Eq. (11) the conditions of a small non-adiabatic contribution are given as

$$
\begin{aligned}
& \left(\mathcal{E}_{2}(0)-\mathcal{E}_{1}(0)\right) \gg\left(W_{2}-W_{1}\right) \gg \frac{\hbar^{2}}{2 m_{0}} \\
& \times\left|\int_{-\infty}^{\infty} d z \varphi_{n_{z \beta}=1}(z, y) \frac{\partial^{2}}{\partial y^{2}} \varphi_{n_{z \beta}=1}(z, y)\right| .
\end{aligned}
$$

Eq. (21) after using Eqs. (10), (17), (20) obtains the form

$$
\begin{aligned}
\left(\mathcal{E}_{2}(0)-\mathcal{E}_{1}(0)\right) & \gg\left(W_{2}-W_{1}\right) \\
& \gg \frac{\hbar^{2}}{2 m_{0}} \sum_{n=1}^{K}\left[\frac{d}{d y} \widetilde{C}_{n_{z \beta}=1}^{(n)}(y)\right]^{2} .
\end{aligned}
$$

Present study assumes that Eq. (22) is satisfied. It shows that the non-adiabatic contributions are small and can be neglected.

Now using Eqs. (15), (16) in Eq. (14) we have

$$
\begin{aligned}
& \sum_{n=1}^{K} C_{n_{z \beta}}^{(n)}(y) \chi_{n}(x)\left[\frac{\mathcal{E}_{n_{z \beta}}(y)}{E_{0}}+\frac{\Lambda_{1} / \Lambda}{x+d(y) / a_{0}}\right. \\
& \left.+\frac{1}{2 n^{2}}-\frac{|e| E_{p} a_{0}}{E_{0}}\left(x+\frac{\xi(y)}{a_{0}}\right)\right]=0 .
\end{aligned}
$$

Multiplying Eq. (23) by $\chi_{m}(x)$, then integrating over $x$, $\int_{0}^{\infty} d x$, and using Eq. (17), we obtain a system of $K$ linear homogeneous equations for $K$ unknown $C^{(n)}(y)$, for a given $y$, as follows

$$
\begin{aligned}
& C^{(m)}(y)\left[\frac{\mathcal{E}(y)}{E_{0}}+\frac{1}{2 m^{2}}-\frac{|e| E_{p} \xi(y)}{E_{0}}\right] \\
& +\sum_{n=1}^{K} C^{(n)}(y)\left[<m\left|\frac{\Lambda_{1} / \Lambda}{x+d(y) / a_{0}}\right| n>\right. \\
& \left.-\frac{|e| E_{p} a_{0}}{E_{0}}<m|x| n>\right]=0,
\end{aligned}
$$

where a matrix element

$$
<m|f(x)| n>=\int_{0}^{\infty} d x f(x) \chi_{m}(x) \chi_{n}(x) .
$$
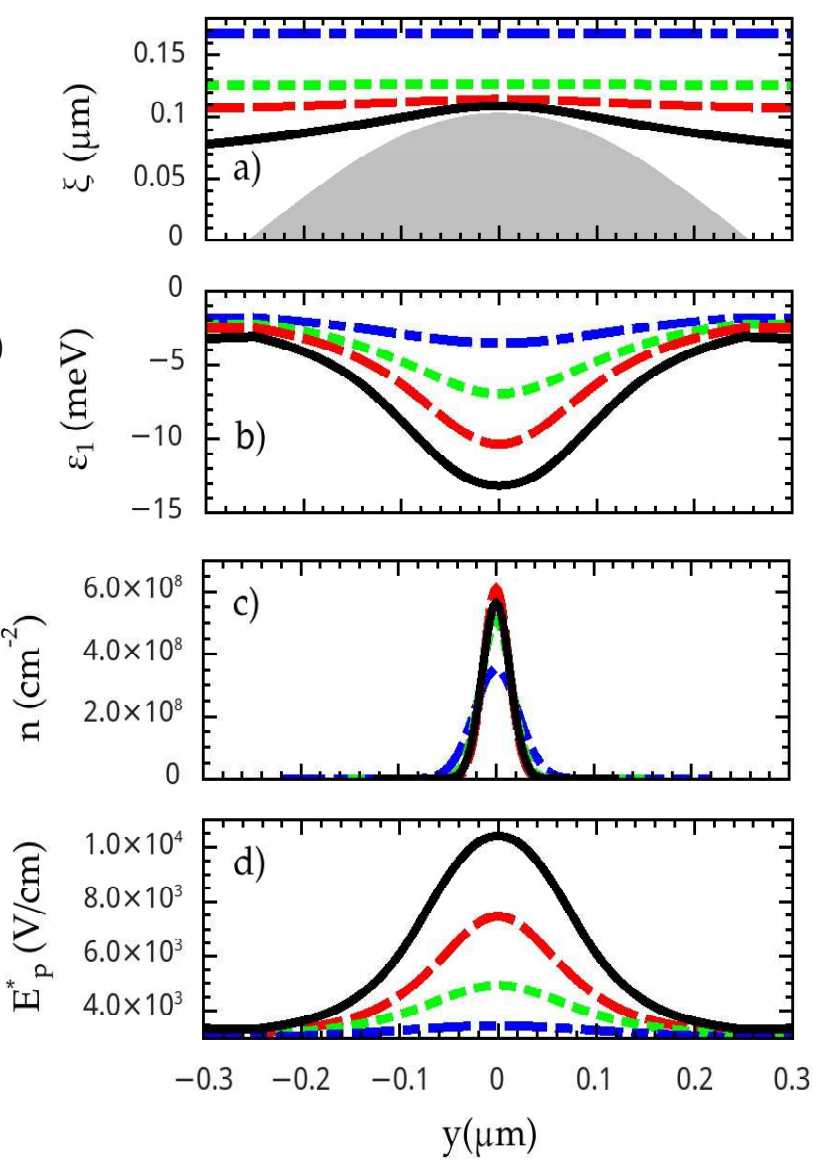

Figure 2: (Color online) By the solid curves, for $H=10 \mathrm{~cm}$, the dashed curves, for $H=2.5 \mathrm{~cm}$, the dotted curves, for $H=0.5 \mathrm{~cm}$, and the dot-dashed curves, for $H=0.05 \mathrm{~cm}$, are plotted on panel: (a) the spatial profile of LH surface $\xi$ (the substrate is shown by a grey background color); (b) the spatial dependence of the ground state energy $\mathcal{E}_{1}$, due to transverse quantization; (c) the spatial dependence of the surface electron density $n$; (d) the spatial dependence of the effective electric field $E_{p}^{*}$ on an electron, in the ground state $\mathcal{E}_{1}$. Here we have $h_{1}=0.1 \mu \mathrm{m}, a=1.0 \mu \mathrm{m}, \Delta L_{y}=1 \mu \mathrm{m}$. For Figs. 2 - $11 T=0.6 \mathrm{~K}, \varepsilon_{S}=5, n_{L}=2 \times 10^{3} \mathrm{~cm}^{-1}$, and $E_{p}=5 \mathrm{~V} / \mathrm{cm}$ are common. $z=-H$ is the level of bulk LH.

To solve a system of equations Eq. (24), we assume a finite value $K_{0}$ for the positive integer $K$. Then Eq. (24) presents a system $K_{0}$ linear homogeneous equations over $K_{0}$ unknown $C^{(n)}(y)$ which will give nontrivial solution Eq. (15) only if the determinant of Eq. (24) is nullified. The latter condition will give $K_{0} \operatorname{roots} \mathcal{E}_{n_{z \beta}}(y)$ that present energies of $K_{0}$ lowest levels due to quantization along $z$, for a given $y$. For each such root $\mathcal{E}_{n_{z \beta}}(y)$ we have a set of $K_{0}$ amplitude functions $C_{n_{z \beta}}^{(n)}(y)$, with $n=1, \ldots, K_{0}$. If we, e.g., increase two times the value of $K_{0}$ then the number of obtained energy levels also will increase two times as "older" levels will be obtained now with higher precision.

In particular, for $K_{0}=2$ assuming $d(y) / a_{0}=4, \varepsilon_{S}=5$ (then $\Lambda_{1} / \Lambda \approx 24.0$ ), $E_{p}=0$ we obtain $<1|x| 1>=1.5$, $<2|x| 2>=6.0,<1|x| 2>=<2|x| 1>=-0.55870,<$ $1|24 /(x+4)| 1>=4.4615,<2|24 /(x+4)| 2>=2.56528$, 

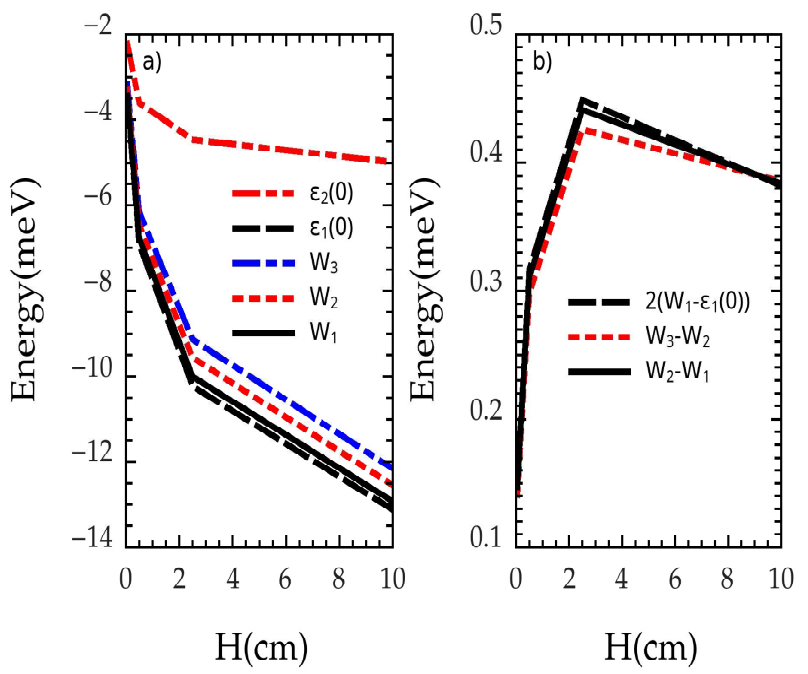

Figure 3: (Color online) The energy spectra are shown as functions of $H$ for conditions of Fig. 2 On panel (a) the solid, the dotted, and the dash-dot curves plot three the lowest levels of the lateral quantization $W_{1}, W_{2}$, and $W_{3}$. The dashed curve and the dot-dotdash curve plot $\mathcal{E}_{1}(0)$ and $\mathcal{E}_{2}(0)$, i.e., two the lowest levels of the transverse quantization at $y=0$. On panel (b) the solid, the dashed, and the dotted curves plot $\left(W_{2}-W_{1}\right), 2\left(W_{1}-\mathcal{E}_{1}(0)\right)$, and $\left(W_{3}-W_{2}\right)$. As these three curves are very close, in an actual region the effective lateral potential $\mathcal{E}_{1}(y)$ for $1 \mathrm{DES}$ is very close to a parabolic one.

$<1|24 /(x+4)| 2>=<2|24 /(x+4)| 1>=0.335448$ it follows for the ground state level $\mathcal{E}_{1}(y) / E_{0}=-5.010008$ (for a bulk LH $\left.\mathcal{E}_{1} / E_{0}=-0.5\right)$ and for the first exited level $\mathcal{E}_{2}(y) / E_{0}=-2.64177$ (for a bulk LH $\mathcal{E}_{2} / E_{0}=-0.125$ ).

\subsection{Self-consistent liquid helium film with a low-dimensional electron system suspended on a substrate}

Now we present the rest of our self-consistent model. Following Ref. [14] we obtain that a profile, $\xi(y)$, of LH suspended on the substrate, $z=h(y)$, is defined by the nonlinear differential equation

$$
\begin{aligned}
& \frac{d^{2} \xi}{d y^{2}}-\left\{\frac{g \sigma}{\alpha_{S T}}\left[\xi(y)+H+\frac{|e| n(y) E_{p}^{*}(y)}{g \sigma}\right]\right. \\
& \left.-\frac{\gamma}{\alpha_{S T}[\xi(y)-h(y)]^{3}}\right\}\left[1+\left(\frac{d \xi}{d y}\right)^{2}\right]^{3 / 2}=0
\end{aligned}
$$

where $H>0$ defines the level of bulk LH as $z=-H$, $\sigma=0.145 \mathrm{~g} / \mathrm{cm}^{3}$ is the helium density, $\gamma=9.5 \times 10^{-15} \mathrm{erg}$ is the vdW coupling constant helium-substrate, $\alpha_{S T}=$ $0.378 \mathrm{erg} / \mathrm{cm}^{2}$ is the surface tension of the liquid helium, and $g$ is the gravity acceleration. Further, we assume that only the fundamental quantum state $n_{z \beta}=1$ can be occupied, at any $y$, and that resulting 2DES (or 1DES) over LH is non-degenerate. Then the electronic density per unit area $n(y)$ obtains the form

$$
\begin{aligned}
n(y)= & \frac{2}{L_{x}} \sum_{k_{x \beta}, n_{y \beta}} e^{\left(\zeta-\widetilde{W}_{1, n_{y \beta}}-\frac{\hbar^{2} k_{x \beta}^{2}}{2 m_{0}}\right) / k_{B} T}\left|\Phi_{n_{y \beta}}(y ; 1)\right|^{2} \\
= & \left(\frac{2 m_{0} k_{B} T}{\pi \hbar^{2}}\right)^{1 / 2} \sum_{n_{y \beta}} e^{\left(\zeta-\widetilde{W}_{1, n_{y \beta}}\right) / k_{B} T} \\
& \times\left|\Phi_{n_{y \beta}}(y ; 1)\right|^{2}
\end{aligned}
$$

where $\zeta$ is the chemical potential, the factor 2 takes into account the spin degeneracy of the energy. Further, the effective electric field, $E_{p}^{*}(y)$, is given as

$$
\begin{aligned}
E_{p}^{*}(y)= & E_{p}+\frac{\Lambda}{|e| a_{0}^{2}} \int_{0}^{\infty} \frac{d x}{x^{2}}\left[\widetilde{\varphi}_{1}^{y}(x)\right]^{2} \\
& +\frac{\Lambda_{1}}{|e| a_{0}^{2}} \int_{0}^{\infty} \frac{d x}{\left[x+d(y) / a_{0}\right]^{2}}\left[\widetilde{\varphi}_{1}^{y}(x)\right]^{2}
\end{aligned}
$$
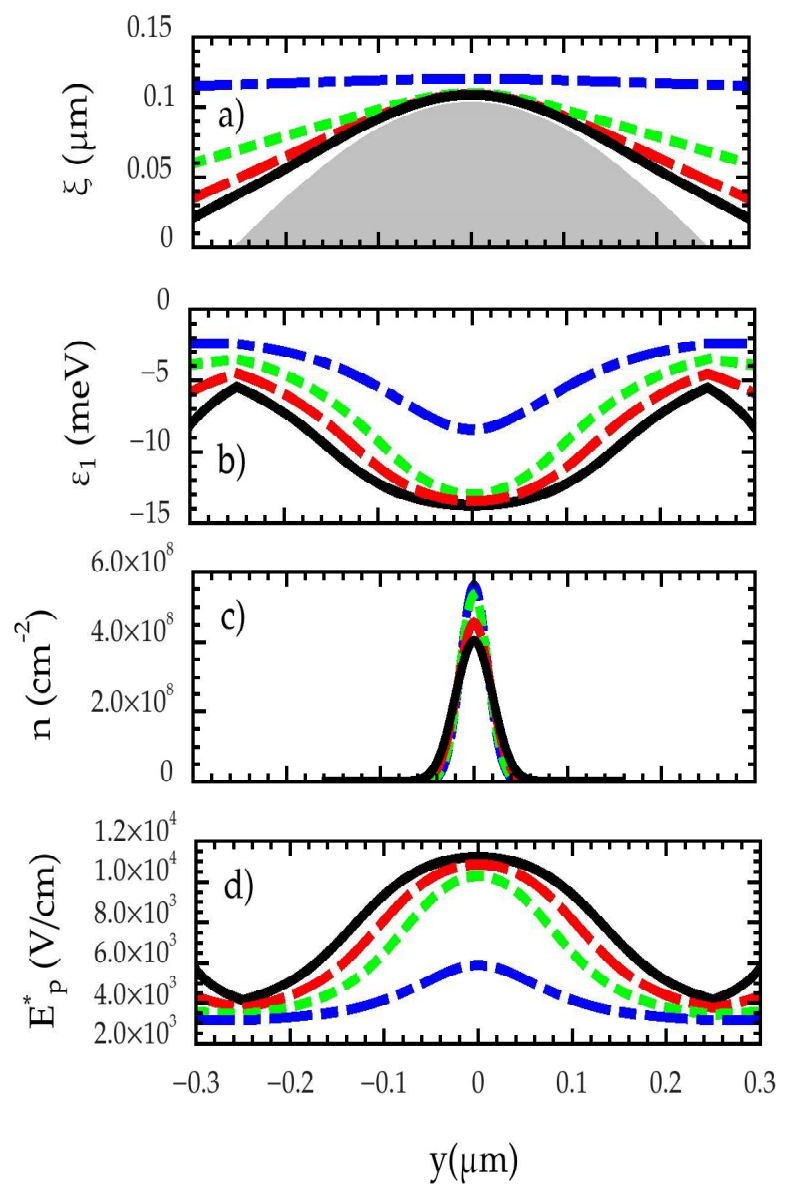

Figure 4: (Color online) Same dependences as in Fig. 2 for the same conditions except $\Delta L_{y}=10 \mu \mathrm{m}$.

The electron area density Eq. (27) is obtained by integration of the bulk microscopic electron density $n_{b u}(y, z)$ 

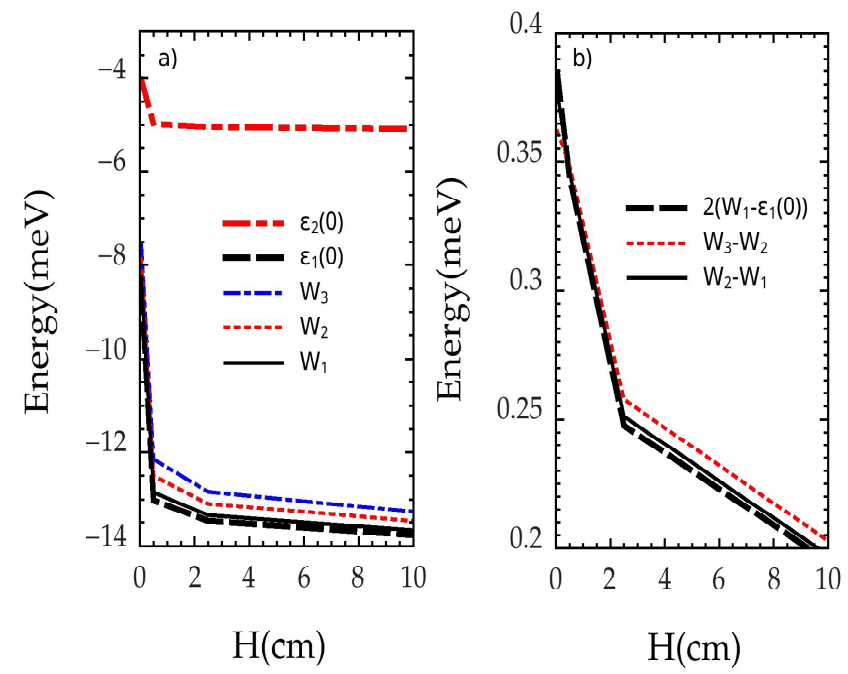

Figure 5: (Color online) The same energy spectra as in Fig. 3 for conditions of Fig. 4] Three curves on panel (b) are very close, same as in Fig. 3(b). It shows that in an actual region the effective lateral potential $\mathcal{E}_{1}(y)$ for 1 DES is very close to a parabolic one.

over $z$ within a region of localization along $z$ of the probability distribution $\varphi_{1}^{2}(z, y)$ and taking into account of Eq.(9). By integrating of Eq. (27) over $y$, from $-\Delta L_{y} / 2$ to $\Delta L_{y} / 2$, and dividing the result by $\Delta L_{y}$ we have the average electron density within the main super cell as

$$
\bar{n}=\frac{1}{\hbar \Delta L_{y}}\left(\frac{2 m_{0} k_{B} T}{\pi}\right)^{1 / 2} \sum_{n_{y \beta}} e^{\left(\zeta-\widetilde{W}_{1, n_{y \beta}}\right) / k_{B} T} .
$$

Then the total number of electrons within the main super cell $N_{\text {tot }}=\bar{n} L_{x} \Delta L_{y}$ and the linear density within a super cell is given as

$$
n_{L}=\frac{1}{\hbar}\left(\frac{2 m_{0} k_{B} T}{\pi}\right)^{1 / 2} \sum_{n_{y \beta}} e^{\left(\zeta-\widetilde{W}_{1, n_{y \beta}}\right) / k_{B} T} .
$$

If in the main region potential Eq. (3) is independent of $y$ then any $\mathcal{E}_{n_{z \beta}}$ also become independent of $y$ and from Eqs. (7), (12) it follows that instead of $n_{y \beta}$ we can use the wave number $k_{y \beta}$. Then, e.g., for the fundamental energy level $\mathcal{E}_{1}<0$ we have that $\Phi_{k_{y \beta}}(y ; 1)=e^{i k_{y \beta} y} / \sqrt{L_{y}}$, and $\widetilde{W}_{1, k_{y}}=\mathcal{E}_{1}+\hbar^{2} k_{y \beta}^{2} / 2 m_{0}$. Then from Eq. (27) it follows $n(y)=\bar{n}$ and

$$
n=\frac{m_{0} k_{B} T}{\pi \hbar^{2}} e^{\left(\zeta+\left|\mathcal{E}_{1}\right|\right) / k_{B} T},
$$

where $e^{\left(\zeta+\left|\mathcal{E}_{1}\right|\right) / k_{B} T} \ll 1$, as electrons are nondegenerate. Point out these conditions correspond to $h_{1}=0$, for the present model of substrate, Eq. (11). Notice that here we have self-consistent Eqs. (15), (24), (26), (28), (31) that define $\mathcal{E}_{1}$ (along with relevant wave function, used in Eq. (28) $), \xi, E_{p}^{*}$, and $\zeta$ para given $H, n$ (or $\left.n_{L}\right), E_{p}$, and $T$. It is seen that even for this rather simple problem (e.g., Eq. (26) reduces to an algebraic equation) the self-consistent set of Eqs. (15), (24), (26), (28), (31) typically will not allow analytical solution.

It is seen that a self-consistent problem becomes a lot more complex if a substrate profile $h(y)$, Eq. (1), parameters, $h_{1}$ and $a$, are finite. Below we study this problem assuming a finite $\Delta L_{y}$ as well. For Eq. (26) two boundary conditions, imposed at the boundaries of the main super cell, obtain the form

$$
d \xi\left( \pm \Delta L_{y} / 2\right) / d y=0
$$
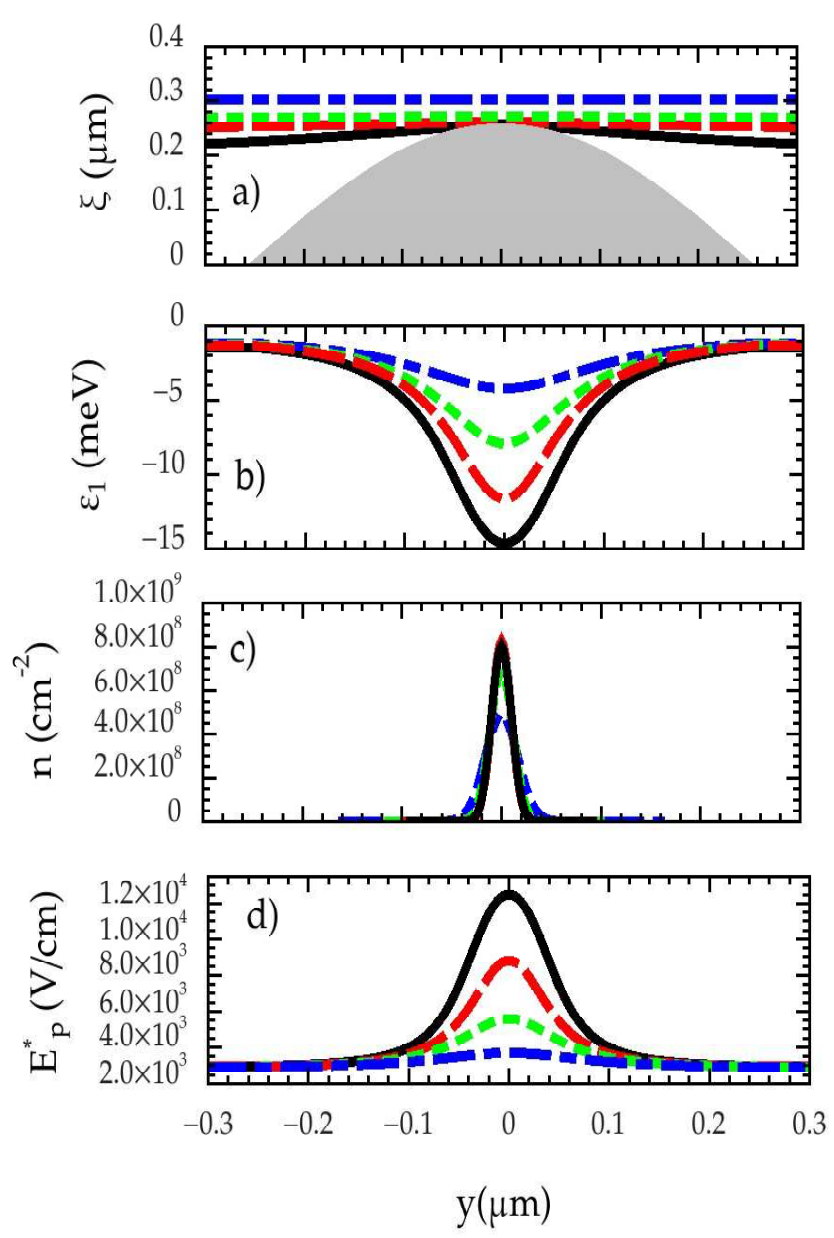

Figure 6: (Color online) Same dependences as in Fig. 2] for the same conditions apart from $h_{1}=0.25 \mu \mathrm{m}$; notice, here $\Delta L_{y}=1 \mu \mathrm{m}$.

\section{One-dimensional electron systems in self-consistent quantum wires suspended on dielectric substrates: Results and discussion}

In below study it is assumed that $T=0.6 \mathrm{~K}, \varepsilon_{S}=5$, $n_{L}=2 \times 10^{3} \mathrm{~cm}^{-1}$, and $E_{p}=5 \mathrm{~V} / \mathrm{cm}$. These conditions are used in Figs. 2 - 11. In addition, in Figs. 2]-11 different 


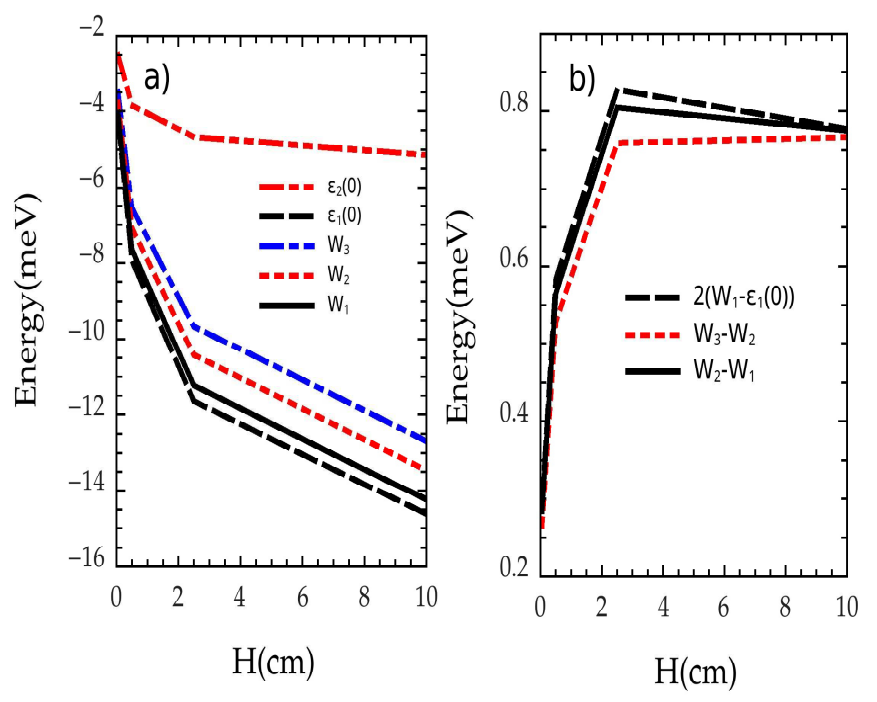

Figure 7: (Color online) The same energy spectra as in Fig. 3 for conditions of Fig. 6. Three curves on panel (b) are close, similar with Fig. 3(b). It shows that in an actual region the effective lateral potential $\mathcal{E}_{1}(y)$ for 1 DES is well approximated by a parabolic one.

substrate profiles $h(y)$, Eq. (1), are used; with different sets of a finite $h_{1}, a$, and $\Delta L_{y}$. Then we need to solve selfconsistent Eqs. (12), (15), (24), (26)-(28), (30). Here, e.g., Eq. (12) and Eq. (26) are mutually coupled the second order nonlinear differential equations. In this self-consistent coupling also Eqs.(15), (24), (27) -(28), (20) are involved. For a given $H$ we solve Eqs. (12), (15), (24), (26) $-(28),(30)$ by using a self-consistent numerical approach. Notice, this problem can not be solved analytically.

First, in Fig. 2 it is assumed the substrate profile $h(y)$ with $h_{1}=0.1 \mu \mathrm{m}, a=1 \mu \mathrm{m}$, and $\Delta L_{y}=1 \mu \mathrm{m}$. We present: in Fig. 2(a) the self-consistent profile $\xi(y)$ of LH surface; in Fig. 2(b) the ground state energy $\mathcal{E}_{1}(y)$ of transverse, mainly, quantization; in Fig. 2(c) the surface electron density $n(y)$, for obtained 1DES; in Fig. 2(d) the effective electric field $E_{p}^{*}(y)$ on an electron in the ground state $\mathcal{E}_{1}(y)$. Point out that in Fig. 2 only $60 \%$ (i.e., $|y| \leq 0.3 \mu \mathrm{m}$ ) of the main super cell are shown to present more clearly the main features.

In Fig. 3 we present the energy spectra as functions of $H$, within the region $10 \mathrm{~cm} \geq H \geq 0.05 \mathrm{~cm}$, for conditions of Fig. 2. In Fig. 3(a) the dashed and the dot-dot-dashed curves show that the gap between two the lowest levels due to mainly transverse quantization is large enough to warrant a two dimensional behavior of electrons even for $H=$ $0.05 \mathrm{~cm}$ as here $\left(\mathcal{E}_{2}(0)-\mathcal{E}_{1}(0)\right) / k_{B} \approx 15.2 \mathrm{~K} \gg T=0.6 \mathrm{~K}$; for $H=0.5 \mathrm{~cm}$ we obtain $\left(\mathcal{E}_{2}(0)-\mathcal{E}_{1}(0)\right) / k_{B} \approx 38.6 \mathrm{~K}$. In Fig. 3(b) the solid curve show that the gap between the lowest two levels due to mainly lateral quantization is large enough to warrant a one dimensional behavior of electrons even for $H=0.05 \mathrm{~cm}$ as here $\left(W_{2}-W_{1}\right) / k_{B} \approx 1.71 \mathrm{~K}$ and $\exp \left(-\left(W_{2}-W_{1}\right) / k_{B} T\right) \approx 5.8 \times 10^{-2}$; for $H=0.5 \mathrm{~cm}$ it fol-
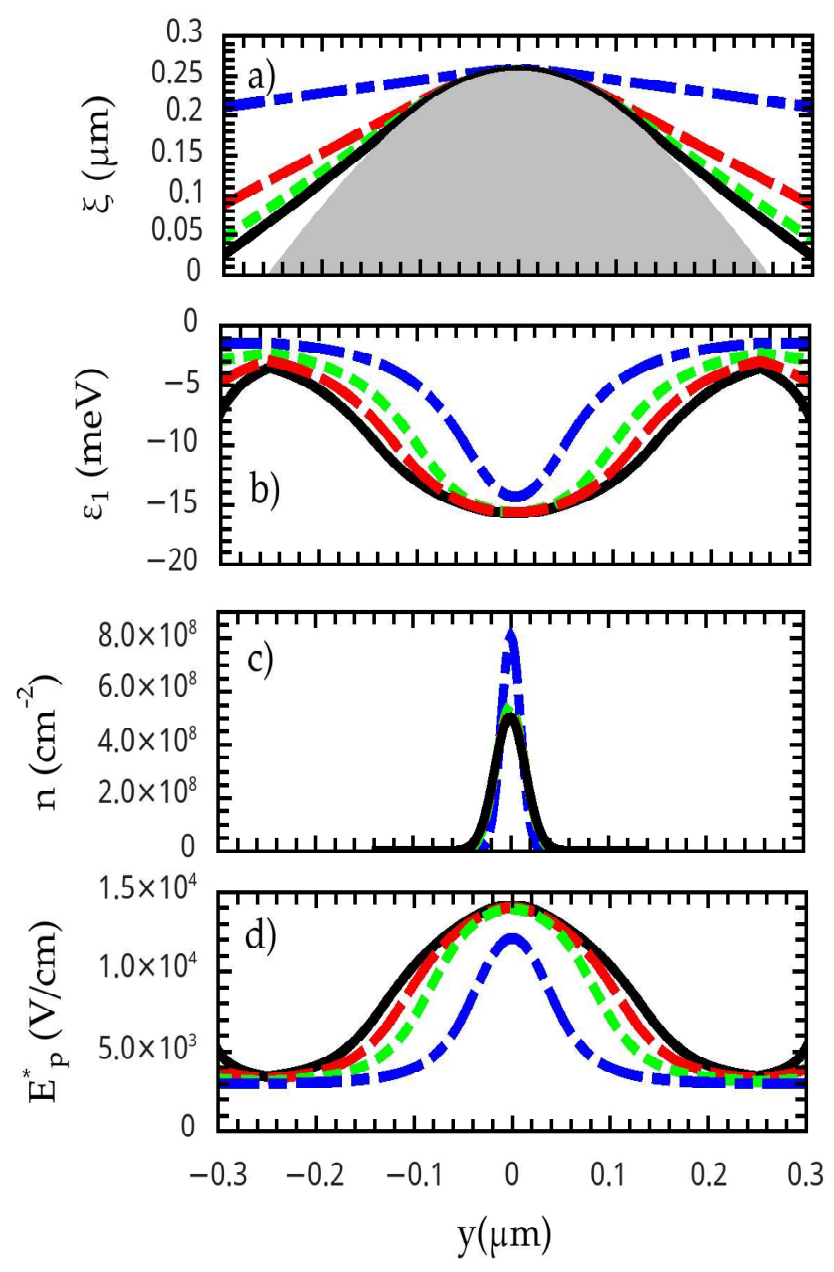

Figure 8: (Color online) Same dependences as in Fig. [6] for the same conditions apart from $\Delta L_{y}=10 \mu \mathrm{m}$.

lows $\left(W_{2}-W_{1}\right) / k_{B} \approx 3.60 \mathrm{~K}$ and $\exp \left(-\left(W_{2}-W_{1}\right) / k_{B} T\right) \approx$ $2.5 \times 10^{-3}$.

In Fig. 3(b) the dashed and the dotted curves plot $2\left(W_{1}-\mathcal{E}_{1}(0)\right)$, and $\left(W_{3}-W_{2}\right)$. As they are very close to the solid curve, plotting $\left(W_{2}-W_{1}\right)$, it shows that for actual regions of energy and $y$ the effective lateral potential $\mathcal{E}_{1}(y)$ can be well approximated by a parabolic potential. For $H=2.5 \mathrm{~cm}$ the gap $\left(W_{2}-W_{1}\right) / k_{B} \approx 5.12 \mathrm{~K}$, i.e., further essentially increases. However, for larger $H=10 \mathrm{~cm}$ the gap shows a decrease, $\left(W_{2}-W_{1}\right) / k_{B} \approx 4.45 \mathrm{~K}$. Point out that the results of Fig. 3 are in a good agreement with Fig. 2. In particular, from Fig. 2(c) it is seen that a density profile, $n(y)$, peak becomes higher and narrower as $H$ grows from $H=0.05 \mathrm{~cm}$ to $H=2.5 \mathrm{~cm}$. However, for $H=10 \mathrm{~cm}$ the peak decreases and widens, in comparison with the one for $H=2.5 \mathrm{~cm}$. This qualitatively is well explained by the LH profiles behavior on Fig. 2(a). Indeed, for $H=10 \mathrm{~cm}$ the $\mathrm{LH}$ profile is essentially closer to the substrate, as $|y|$ grows from 0 , in a wider lateral region than for $H=2.5 \mathrm{~cm}$. It leads to more soft lateral confine- 

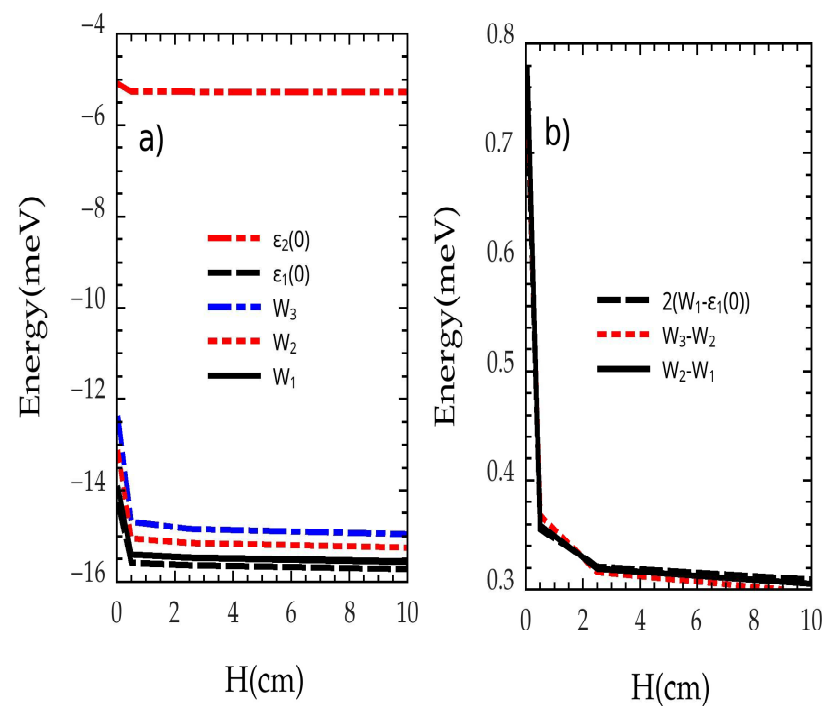

Figure 9: (Color online) The same energy spectra as in Fig. 7 for conditions of Fig. 8 Three curves on panel (b) are close, similar with Fig. 7 b). This shows that in an actual region the effective lateral potential $\mathcal{E}_{1}(y)$ for $1 \mathrm{DES}$ is very well approximated as a parabolic one.

ment as it is seen also from Fig. 2(b). Point out that for $H=0.05 \mathrm{~cm}, 0.5 \mathrm{~cm}, 2.5 \mathrm{~cm}$ and $10 \mathrm{~cm}$ Fig. 2(c) and Fig. 2(b) show that the peaks of Fig. 2(c) are well approximated by the Gaussian, $n(y) \propto \ell_{y}^{-1} \times \exp \left(-y^{2} / \ell_{y}^{2}\right)$, with $\ell_{y} \lesssim 32 \mathrm{~nm}, 22 \mathrm{~nm}, 18 \mathrm{~nm}$ and $20 \mathrm{~nm}$, respectively. In particular, it follows that the maximum density $n(0) \propto \ell_{y}^{-1}$ and $\ell_{y} \propto\left(W_{2}-W_{1}\right)^{-1 / 2}$. In addition, Figs. 2(b), 2(d) show that transverse confinement of 1DES becomes stronger as $H$ grows, and the effective electric field, $E_{p}^{*}(y)$, increases.

In Fig. 4 we present the same dependences as in Fig. 2 assuming the same parameters as in Fig. 2] except of $\Delta L_{y}=10 \mu \mathrm{m}$. Point out that in Fig. 4 only a small part, $|y| \leq 0.3 \mu \mathrm{m}$, of the main super cell, $|y| \leq 5 \mu \mathrm{m}$, is shown to present more clearly the main features. In Fig. [5 we present the same energy spectra as in Fig. 3 as functions of $H$, at $10 \mathrm{~cm} \geq H \geq 0.05 \mathrm{~cm}$, for conditions of Fig. 4. In Fig. 5(a) the dashed and the dot-dot-dashed curves show that the gap between the lowest two levels due to mainly transverse quantization is large enough to warrant a two dimensional behavior of electrons. Indeed, for $H=0.05 \mathrm{~cm}$ it follows that $\left(\mathcal{E}_{2}(0)-\mathcal{E}_{1}(0)\right) / k_{B} \approx 51.2 \mathrm{~K}$ and with an increase of $H$ the gap further grows; it keeps close to $90 \mathrm{~K}$ for $H \geq 0.5 \mathrm{~cm}$.

In Fig. 5(b) the solid curve show that the gap between the lowest two levels due to mainly lateral quantization is large enough to warrant a one dimensional behavior of electrons for $H=0.05 \mathrm{~cm}$ as here $\left(W_{2}-W_{1}\right) / k_{B} \approx 4.37 \mathrm{~K}$ and $\exp \left(-\left(W_{2}-W_{1}\right) / k_{B} T\right) \approx 6.8 \times 10^{-4}$. For growing $H$ it follows that $\left(W_{2}-W_{1}\right)$ decreases. However, it is still large enough to warrant a one dimensional behavior of electrons. In addition, in Fig. 5(b) we observe that the
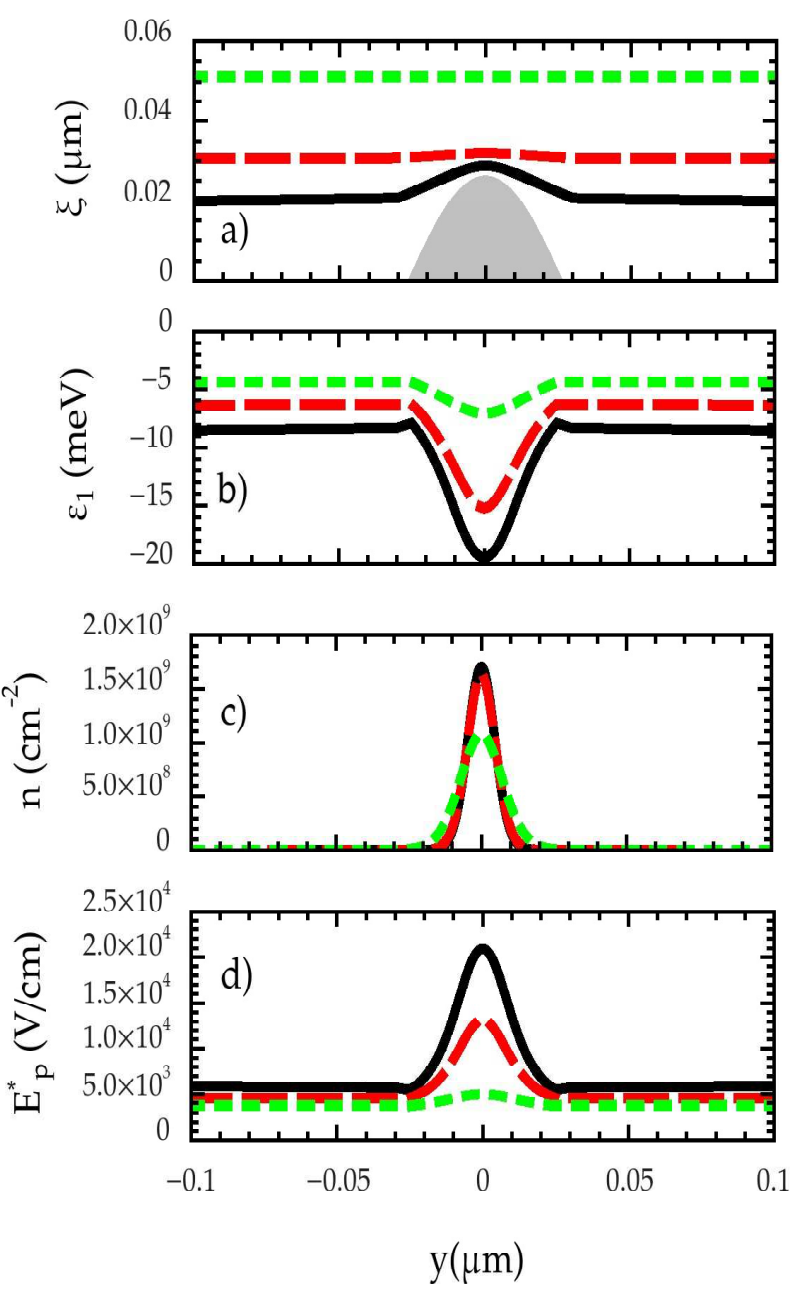

Figure 10: (Color online) Same dependences as in Fig. 2 or Fig. 6 for the same conditions except for: $h_{1}=0.025 \mu \mathrm{m}, a=0.1 \mu \mathrm{m}$. In addition, the curves for $H=0.05 \mathrm{~cm}$ are absent.

solid, the dashed, and the dotted curves are very close. This shows that for actual regions of energy and $y$ the effective lateral potential $\mathcal{E}_{1}(y)$ can be well approximated by a parabolic potential. In agreement with Fig. [5)(b), in Fig. 4(c) a density profile, $n(y)$, peak becomes lower and wider as $H$ grows from $H=0.05 \mathrm{~cm}$ to $H=10 \mathrm{~cm}$. This qualitatively is well explained by the LH profiles behavior on Fig. 4(a). Indeed, due to larger $\Delta L_{y}$ in Fig. 4 than in Fig. 2 a $\mathrm{LH}$ profile is essentially closer to the substrate within an actual lateral region, as $|y|$ grows from 0 , and this region is essentially wider, for the same $H$, than in Fig. 2 (a). Point out that qualitatively similar dependence on $H$ holds in Figs. 2, 3 only as $H$ grows from $H=2.5 \mathrm{~cm}$ to $H=10 \mathrm{~cm}$. The peaks of Fig. 4(c) are well approximated by the Gaussian, $n(y) \propto \ell_{y}^{-1} \times \exp \left(-y^{2} / \ell_{y}^{2}\right)$, with $\ell_{y} \approx$ $20 \mathrm{~nm}, 21 \mathrm{~nm}, 25 \mathrm{~nm}$ and $28 \mathrm{~nm}$ for $H=0.05 \mathrm{~cm}, 0.5 \mathrm{~cm}$, $2.5 \mathrm{~cm}$ and $10 \mathrm{~cm}$, respectively.

In Fig. 6 we present the same dependences as in Fig. 2 assuming the same parameters as in Fig. 2 apart from $h_{1}=0.25 \mu \mathrm{m}$. Point out that in Fig. 6 only a part, $|y| \leq 0.3 \mu \mathrm{m}$, of the main super cell, $|y| \leq 0.5 \mu \mathrm{m}$, is shown to present more clearly the main features. In Fig. 7 we 


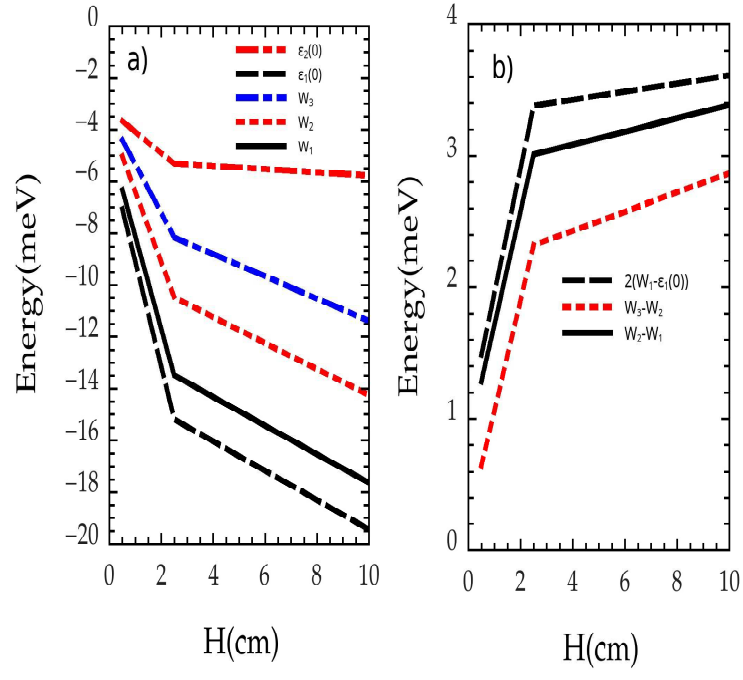

Figure 11: (Color online) The same energy spectra as in Fig. 7 are plotted for conditions of Fig. 10 here $10 \mathrm{~cm} \geq H \geq 0.5 \mathrm{~cm}$

present the same energy spectra as in Fig. 3 as functions of $H$, at $10 \mathrm{~cm} \geq H \geq 0.05 \mathrm{~cm}$, for conditions of Fig. 6. Figs. 6. 7 show qualitatively the same behavior as Figs. 2. 3. In particular, Fig. 7(b) shows the same qualitative behavior as Fig. 3(b). Increase in the height of modulation of the substrate from $h_{1}=0.1 \mu \mathrm{m}$ to $h_{1}=0.25 \mu \mathrm{m}$ leads to higher electronic concentration in the center of the channel, $n(0)$. Then, e.g., considering $H=10 \mathrm{~cm}$, the minimum LH film thickness, $d(0)=\xi(0)-h_{1}$, decreases from $9.4 \mathrm{~nm}$, for $h_{1}=0.1 \mu \mathrm{m}$, to $7.5 \mathrm{~nm}$, for $h_{1}=0.25 \mu \mathrm{m}$.

Further, in Fig. 77(a) the dashed and the dot-dotdashed curves show that the gap between two the lowest levels due to mainly transverse quantization is large enough to warrant a two dimensional behavior of electrons. Indeed, for $H=0.05 \mathrm{~cm}$ and $0.5 \mathrm{~cm}$ it follows that $\left(\mathcal{E}_{2}(0)-\right.$ $\left.\mathcal{E}_{1}(0)\right) / k_{B} \approx 19.5 \mathrm{~K}$ and $47.5 \mathrm{~K}$. With an increase of $H$ the gap further grows. In Fig. 7(b) the solid curve show that the gap between the lowest two levels due to mainly lateral quantization is large enough to warrant a one dimensional behavior of electrons even for $H=0.05 \mathrm{~cm}$ as here $\left(W_{2}-\right.$ $\left.W_{1}\right) / k_{B} \approx 3.29 \mathrm{~K}$ and $\exp \left(-\left(W_{2}-W_{1}\right) / k_{B} T\right) \approx 4.15 \times$ $10^{-3}$. For $H=0.5 \mathrm{~cm}$ it follows that $\left(W_{2}-W_{1}\right) / k_{B} \approx$ $6.55 \mathrm{~K}$ and $\exp \left(-\left(W_{2}-W_{1}\right) / k_{B} T\right) \approx 1.8 \times 10^{-5}$. Point out that in Fig. 6(c) a density profile peak is well approximated as the Gaussian, $n(y) \propto \ell_{y}^{-1} \times \exp \left(-y^{2} / \ell_{y}^{2}\right)$, with $\ell_{y}$ decreasing from $23 \mathrm{~nm}$ to $16 \mathrm{~nm}$, and $14 \mathrm{~nm}$ as $H$ grows from $0.05 \mathrm{~cm}$ to $0.5 \mathrm{~cm}$, and $2.5 \mathrm{~cm}$.

In Fig. 8 we present the same dependences as in Fig. 6] assuming the same parameters as in Fig. 6] except for $\Delta L_{y}=10 \mu \mathrm{m}$. Point out that in Fig. 8 only a small part, $|y| \leq 0.3 \mu \mathrm{m}$, of the main super cell, $|y| \leq 5 \mu \mathrm{m}$, is shown. In Fig. 9 we present the same energy spectra as in Fig. 7 as functions of $H$, at $10 \mathrm{~cm} \geq H \geq 0.05 \mathrm{~cm}$, for conditions of Fig. 8. Point out that Figs. 8, 9] show qualitatively the same behavior as Figs. 4, 5. Increase in a height of substrate modulation from $h_{1}=0.1 \mu \mathrm{m}$ to $h_{1}=0.25 \mu \mathrm{m}$ leads to higher electronic concentration in the center of the channel, $n(0)$. Then, considering $H=10 \mathrm{~cm}$, the minimum LH film thickness, $d(0)$, decreases from $8.5 \mathrm{~nm}$, for $h_{1}=$ $0.1 \mu \mathrm{m}$, in Fig. 4(a) to $6.4 \mathrm{~nm}$, for $h_{1}=0.25 \mu \mathrm{m}$, in Fig. 8(a).

In Fig. 9(a) the dashed and the dot-dot-dashed curves show that the gap between the lowest two levels due to mainly transverse quantization is large enough to warrant a two dimensional behavior of electrons. Indeed, for $H=$ $0.05 \mathrm{~cm}$ it follows that $\left(\mathcal{E}_{2}(0)-\mathcal{E}_{1}(0)\right) / k_{B} \approx 107 \mathrm{~K}$ and for $H \geq 0.5 \mathrm{~cm}$ the gap increases a bit and becomes $\approx 120 \mathrm{~K}$. In Fig. 9(b) the solid curve shows that the gap between the lowest two levels due to mainly lateral quantization is large enough to warrant a one dimensional behavior of electrons for $H=0.05 \mathrm{~cm}$ as here $\left(W_{2}-W_{1}\right) / k_{B} \approx 9.02 \mathrm{~K}$ and $\exp \left(-\left(W_{2}-W_{1}\right) / k_{B} T\right) \approx 2.9 \times 10^{-7}$. In addition, even though $\left(W_{2}-W_{1}\right)$ decreases for growing $H$ it is still large enough to warrant a one dimensional behavior of electrons. In agreement with this, in Fig. 8(c) a density profile peak is the narrowest and the highest for $H=0.05 \mathrm{~cm}$, and it becomes wider and lower as $H$ grows. These peaks are well approximated by the Gaussian, $n(y) \propto \ell_{y}^{-1} \times \exp \left(-y^{2} / \ell_{y}^{2}\right)$, with $\ell_{y} \approx 13 \mathrm{~nm}$ for $H=0.05 \mathrm{~cm}$ and $\ell_{y} \approx 20 \mathrm{~nm}$ for $H \geq$ $0.5 \mathrm{~cm}$.

In Figs. 10 and 11 we present the same dependences as in Figs. 6, 7 assuming, except for $h_{1}=0.025 \mu \mathrm{m}$, $a=0.1 \mu \mathrm{m}$ and $10 \mathrm{~cm} \geq H \geq 0.5 \mathrm{~cm}$, the same parameters. I.e., in comparison with Figs. 6, 7] in Figs. 10, 11 the parameters $h_{1}, a$ are reduced by ten times as the super cell size is kept constant, at $\Delta L_{y}=1 \mu \mathrm{m}$. Point out that in Fig. 10 only a small part, $|y| \leq 0.1 \mu \mathrm{m}$, of the main super cell, $|y| \leq 0.5 \mu \mathrm{m}$, is shown. Fig. 10 shows qualitatively similar behavior with Fig. 6 for $H=0.5,2.5$, and $10 \mathrm{~cm}$. In particular, in Fig. 11(a) the dashed and the dot-dot-dashed curves show that the gap between two the lowest levels due to mainly transverse quantization is large enough to warrant a two dimensional behavior of electrons. Indeed, for $H=0.5 \mathrm{~cm}$ it follows that $\left(\mathcal{E}_{2}(0)-\mathcal{E}_{1}(0)\right) / k_{B} \approx 39.4 \mathrm{~K}$; with further increase of $H$ the gap grows rapidly.

In Fig. 11(b) the solid (dashed) curve shows that $W_{2}-W_{1}\left(2\left(W_{1}-\mathcal{E}_{1}(0)\right)\right)$ for $H=0.5 \mathrm{~cm}, 2.5 \mathrm{~cm}$, and $10 \mathrm{~cm}$ is given as $14.9 \mathrm{~K}(17.2 \mathrm{~K}), 34.9 \mathrm{~K}(39.2 \mathrm{~K})$, and $39.2 \mathrm{~K}$ $(41.9 \mathrm{~K})$. Here the dotted curve shows that $W_{3}-W_{2}$ is given, respectively, as $7.48 \mathrm{~K}, 26.9 \mathrm{~K}$, and $33.3 \mathrm{~K}$. These shows that for $H \gtrsim 0.5 \mathrm{~cm}$ all essential conditions of our treatment are satisfied. Fig. 11(b) shows that for $H \gtrsim$ $0.5 \mathrm{~cm}$ the gaps between three the lowest levels due to mainly lateral quantization is large enough to warrant a one dimensional behavior of electrons. In particular, for $H=0.5 \mathrm{~cm}$ and $10 \mathrm{~cm}$, we have $\exp \left(-\left(W_{2}-W_{1}\right) / k_{B} T\right) \approx$ $2.7 \times 10^{-11}$ and $\approx 4.2 \times 10^{-29}$. Fig. [10(c) shows that the density profile $n(y)$ peak is well approximated by the Gaussian, $n(y) \propto \ell_{y}^{-1} \times \exp \left(-y^{2} / \ell_{y}^{2}\right)$, with $\ell_{y}$ decreasing from $\approx 10.4 \mathrm{~nm}$ to $\approx 6.6 \mathrm{~nm}$ as $H$ grows from $0.5 \mathrm{~cm}$ to $10 \mathrm{~cm}$. Fig. 10 (c) shows that the peak becomes narrower 
and higher as $H$ grows.

Point out that in present figures a maximum electron density, $n(y=0)$, for the obtained 1DESs is not too high as these 1DESs are non-degenerated. It is in agreement with the assumed conditions. Notice that for QWs studied in Figs. 2 - 11 effect of tunnel coupling between super cells on obtained self-consistent 1DESs, localized at $y=0$, is negligible already for extremely week disorder effects on pertinent energy levels.

\section{Conclusions}

We obtained a strong self-consistent enhancement of the transverse and the lateral quantizations of an electron on LH suspended over the specially modulated dielectric substrates. The enhancement is due to a strong mutual interplay between the transverse and the lateral movements of an electron. It is related also with self-consistent dependences of LH profile and of LH thickness over the substrate. Strong enhancements for the effective electron image potential and electric field are obtained due to a self-consistent modification of LH thickness.

Non-degenerated 1DESs are obtained, at relatively high temperature $T=0.6 \mathrm{~K}$ and rather weak an external electric field $E_{p}=5 \mathrm{~V} / \mathrm{cm}$, for dielectric substrates with different nanoscale modulation. In particular, for $H=0.5 \mathrm{~cm}$ in studied model setups we obtained that the gaps between two the lowest electron states due to the lateral confinement $\left(W_{2}-W_{1}\right) / k_{B}$ appear within the interval from $3.60 \mathrm{~K}$ to $14.9 \mathrm{~K}$ as the gaps between two the lowest electron levels due to the transverse confinement $\left(\mathcal{E}_{2}(0)-\mathcal{E}_{1}(0)\right) / k_{B}$ appear within the interval from $38.6 \mathrm{~K}$ to $120 \mathrm{~K}$.

We demonstrated that in an actual region the effective lateral potential $\mathcal{E}_{1}(y)$ for a $1 \mathrm{DES}$ is very close to a parabolic one. In addition, the electron density is well approximated as $n(y) \propto \ell_{y}^{-1} \times \exp \left(-y^{2} / \ell_{y}^{2}\right)$, with $\ell_{y}$ given in the range from $6.6 \mathrm{~nm}$ to $32 \mathrm{~nm}$. This form of $n(y)$ is qualitatively similar with the probability to find an electron at the fundamental Landau level [26] $\propto \ell_{0}^{-1} \times \exp \left(-y^{2} / \ell_{0}^{2}\right)$, where $\ell_{0}$ is the quantum magnetic length. A strong "longrange" effect of $\Delta L_{y}$ on the properties of a self-consistent 1DES is shown as $\Delta L_{y}$ decrease from $10 \mu \mathrm{m}$ to $1 \mu \mathrm{m}$.

In addition, our study of present non-degenerated 1DESs, at $T=0.6 \mathrm{~K}$, have shown that assumed linear density $n_{L}=2 \times 10^{3} \mathrm{~cm}^{-1}$ is relatively small. In a sense that its influence on obtained LH profile, self-consistent transversal and lateral quantizations, a spatial form of normalized electron density, etc. is very weak. Then we can speculate that present results also will well approximate results for $T<0.6 \mathrm{~K}$. In particular, for $T \ll 1 \mathrm{~K}$, i.e., for temperatures of particular interest for a quantum computer [10, 12].

\section{Acknowledgments}

This work was supported by the Brazilian FAPEAM (Fundação de Amparo à Pesquisa do Estado do Amazonas) Grants: Universal Amazonas (Edital 021/2011), O. G. B..

\section{References}

\section{References}

[1] W. T. Sommer, Phys. Rev. Lett. 12, (1964) 271.

[2] M. Cole and M. H. Cohen, Phys. Rev. Lett. 23, (1969) 1238.

[3] V. B. Shikin, Sov. Phys. JETP 31, (1970) 936.

[4] E. Y. Andrei (Ed.), Two-Dimensional Electron Systems on Helium and other Cryogenic Substrates, Kluwer Academic, Dordrecht, 1997.

[5] Yu. Z. Kovdrya, Low Temp. Phys. 29, (2003) 77.

[6] Y. Monarkha and K. Kono, Two-Dimensional Coulomb Liquids and Solids (Vol. 142, Springer Series in Solid-State Sciences), Springer, Berlin, 2004.

[7] Vl. L. Ginzburg and Yu. P. Monarkha,, Fiz. Nizk. Temp. 4, (1978) 1236 [Sov. J. Low Temp. Phys. 4, (1978) 580 ].

[8] Yu. Z. Kovdrya and Yu. P. Monarkha, Fiz. Nizk. Temp. 12, (1986) 1011 [Sov. J. Low Temp. Phys. 12, (1986) 571 ].

[9] A. M. C. Valkering and R. W. van der Heiden, Physica B 249251, (1998) 652 ; A. M. C. Valkering, J. Klier, E. Teske, R. W. van der Heijden, P. Leiderer, J. Low Temp. Phys. 113, (1998) 1115 .

[10] P. M. Platzman and M. I. Dykman, Science 284, (1999) 1967.

[11] A. J. Dahm, J. M. Goodkind, I. Karakurt, S. Pilla, J. Low. Temp. Phys. 126, (2002) 709.

[12] M. I. Dykman, P. M. Platzman, and P. Seddighrad, Phys. Rev. B 67, (2003) 155402.

[13] A. C. A. Ramos, O. G. Balev, and N. Studart, Phys. Rev. B 70, (2004) 035414.

[14] A. C. A. Ramos, O. G. Balev, and N. Studart, J. Low Temp. Phys. 138, (2005) 403.

[15] S. A. Lyon, Phys. Rev. A 74, (2006) 052338.

[16] G. Sabouret, F. R. Bradbury, S. Shankar, J. A. Bert, and S. A. Lyon, Appl. Phys. Lett. 92, (2008) 082104.

[17] A. C. A. Ramos, A. Chaves, G. A. Farias, and F. M. Peeters, Phys. Rev B 77, (2008) 045415.

[18] D. I. Schuster, A. Fragner, M. I. Dykman, S. A. Lyon, and R. J. Schoelkopf, Phys. Rev. Lett. 105, (2010) 040503.

[19] F. R. Bradbury, Maika Takita, T. M. Gurrieri, K. J. Wilkel, Kevin Eng, M. S. Carroll, and S. A. Lyon, Phys. Rev. Lett. 107, (2011) 266803.

[20] H. Ikegami, H. Akimoto, D. G. Rees, and K. Kono, Phys. Rev. Lett. 109 (2012) 236802.

[21] A. B. Petrin, JETP 116 (3), (2013) 486.

[22] D. S. Dantas, A. Chaves,, G. A. Farias, A. C. A. Ramos, and F. M. Peeters, J. Low Temp. Phys. 173 (2013) 207.

[23] H. Ikegami, H. Akimoto, and K. Kono, J. Low Temp. Phys. 179 (2015) 251.

[24] W. S. Boyle and G. E. Smith, Bell Syst. Tech. J. 49 (1970) 587, http://alcatel-lucent.com.bstj/vol49-1970/articles/bstj494-587.pdf.

[25] A. I. Anselm, Introduction to Semiconductor Theory, Prentice Hall, Englewood Cliffs, NJ, 1982 [A. I. Anselm, Introduction to Semiconductor Theory, Nauka, Moscow, 1978 (in Russian)].

[26] L.D. Landau and E.M. Lifshitz, Quantum Mechanics (NonRelativistic Theory), Elsevier Science Ltd., Third Revised edition, 1977.

[27] I. S. Gradshteyn and I. M. Ryzhik, Table Integrals, Series, and Products, Academic Press, New York, 1980. 